\title{
NEAR CRITICAL FREE SURFACE FLOW PAST AN OBSTACLE*
}

BY

\author{
SUSAN L. COLE \\ Pomona College
}

\begin{abstract}
This paper describes the nonlinear effects produced by arbitrarily small bumps in two-dimensional free surface flows with Fraude numbers close to $1^{+}$. These effects are determined by asymptotically matching (approximate) solutions to the ideal flow equations.
\end{abstract}

1. Introduction. The problem of steady free surface flow is examined in the coordinate frame depicted in Fig. 1. Uniform flow with velocity $U$ and undisturbed height $h$ approaches a small bump from upstream infinity. The shape of the bottom is described by the function $y=\varepsilon f(x)$.

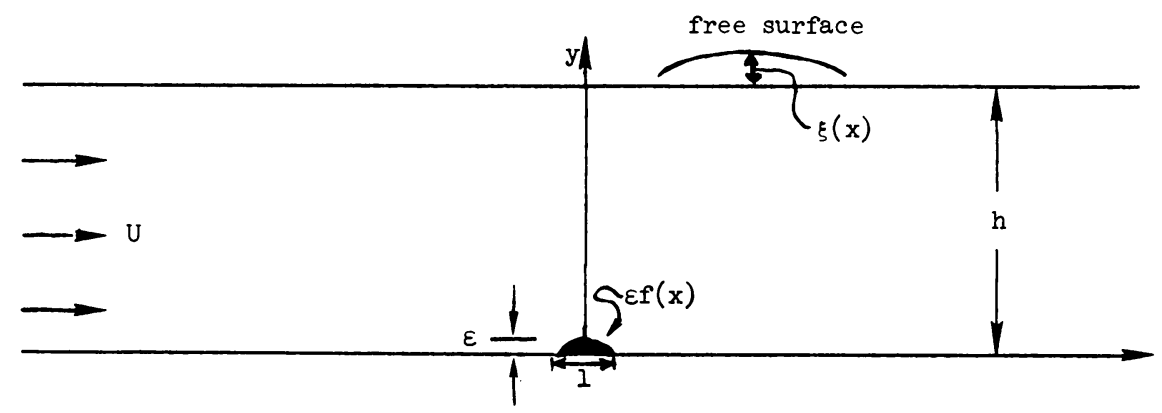

FIG. 1. Coordinate frame of free surface problem.

The motion of an irrotational fluid can be characterized by a velocity potential $\Phi(x, y)$ where the velocity of the fluid at any point is given by $\nabla \Phi$. For a flow which is also incompressible, continuity requires that $\Phi$ satisfies Laplace's equation

$$
\Phi_{x x}+\Phi_{y y}=0 .
$$

The inviscid boundary conditions are that the flow is tangent to the bottom,

$$
\Phi_{y}=\Phi_{x} \varepsilon f_{x} \text { on } y=\varepsilon f(x),
$$

* Received September 13, 1982. The author wishes to thank the Mathematics Branch of the Office of Naval Research for support of this research under contract N00014-80-C-0373 Mod. P00004. 
tangent to the free surface,

$$
\Phi_{y}=\Phi_{x} \xi_{x} \quad \text { on } y=h+\xi(x),
$$

and also satisfies Bernoulli's equation there,

$$
P / \rho+\frac{1}{2}\left(\Phi_{x}^{2}+\Phi_{y}^{2}\right)+g z=\text { const. on } y=h+\xi(x) .
$$

The pressure $P$ is constant along the free surface and the density $\rho$ is constant everywhere.

Equations (1)-(4) along with the boundary conditions that

$$
\Phi \underset{x \rightarrow-\infty}{\rightarrow} U x \text { and } \xi \underset{x \rightarrow-\infty}{\rightarrow} 0
$$

are the basic differential equation and boundary conditions which describe the fluid motion. The relevent nondimensional parameters are the Froude number $F=U / \sqrt{g h}$ and the bump height $\varepsilon$. Flows with $F>1$ are referred to as supercritical, flows with $F<1$ as subcritical flows with $F=1$ as critical. The physical significance of the term critical lies in fact that infinitesimal waves travel at a maximum speed $\sqrt{g h}$ which corresponds to $F=1$.

Equations (1)-(4) are quite difficult to solve for an arbitrary bump due to the nonlinearity of the boundary conditions and the interaction between the free surface location and the fluid velocity. In order to help understand the general solution, it is useful to consider the case of infinitesimally small bumps corresponding to $\varepsilon \rightarrow 0$ with all other quantities fixed.

2. The linear solution. The linear approximation is based on the assumption that the velocity potential and free surface satisfy asymptotic expansions of the form

$$
\begin{aligned}
\Phi(x, y ; \varepsilon) & =U\left\{x+\varepsilon \varphi(x, y)+\varepsilon^{2} \varphi_{2}(x, y)+\cdots\right\}, \\
\xi(x ; \varepsilon) & =\varepsilon \eta(x)+\varepsilon^{2} \eta_{2}(x)+\cdots
\end{aligned}
$$

where the functions $\varphi, \eta, \varphi_{2}, \eta_{2}$ etc. are order 1 and the terms of order $\varepsilon^{2}$ or higher are negligible in comparison to the order $\varepsilon$ terms. Substituting these expansions into equations (1)-(4), expanding $\Phi$ in the boundary equations about the fixed location $y=0$ or $y=h$, and retaining terms of order $\varepsilon$ gives the usual linear relations

$$
\begin{gathered}
\varphi_{x x}+\varphi_{y y}=0, \\
\varphi_{y}=f_{x} \quad \text { on } y=0, \\
\varphi_{y}=\eta_{x} \quad \text { on } y=h,
\end{gathered}
$$

and

$$
F^{2} h \varphi_{x}+\eta=0 \quad \text { on } y=h .
$$

The free surface boundary Eqs. (7) and (8) can be combined to form a single equation which is independent of $\eta$,

$$
F^{2} h \varphi_{x x}+\varphi_{y}=0 \quad \text { on } y=h
$$


The complicated interaction between the velocity potential and the free surface in the nonlinear problem is absent in the linear problem. Kelvin [1] solved the linearized problem exactly.

For $f(x)$ equal to the delta function ${ }^{1} \delta(x)$, the linear solution for the velocity potential is

$$
\varphi=\frac{-i}{2 \pi} \int_{\gamma i-\infty}^{\gamma i+\infty} e^{i k x}\left[\operatorname{sh} k z-\left(\frac{F^{2} k h \operatorname{sh} k h-\operatorname{ch} k h}{F^{2} k h \operatorname{ch} k h-\operatorname{sh} k h}\right) \operatorname{ch} k z\right] d k
$$

The qualitative nature of the free surface as a function of the parameter $F$ can be deduced from the properties of the denominator of the integrand $D(k)=F^{2} k h \mathrm{ch} k h-$ $\operatorname{sh} k h$.

- For $F<1$, the equation $D(k)=0$ has real roots at $k=0$ and $\pm k^{*}$ only. The roots $k= \pm k^{*}$ correspond to the wave number of the free wave solutions and give rise to the wave train that is present in the linear downstream solution for $F<1$. The point $k=0$ is a removable singularity of the integrand for $F<1$.

- For $F>1$, the equation $D(k)=0$ has no nonzero real roots. This corresponds to the fact that there are no free wave solutions for $F>1$ and no wave train in the linear downstream solution. The point $k=0$ is a removable singularity of the integrand for $F>1$ also.

- The integral expression for $\eta$ is infinite for $F=1$ since

$$
D(k) \underset{k \rightarrow 0}{\sim} O\left(k^{3}\right) \text {. }
$$

There is no steady linear solution for $F=1$.

- The equation $D(k)=0$ has an infinite number of purely imaginary roots which are all bounded away from zero for $F$ bounded away from $1^{+}$. As $F$ tends to $1^{+}$, the smallest imaginary root tends to zero and gives rise to a free surface solution which tends to infinity as $F \rightarrow 1^{+}$.

The integral expression for $\eta$ can be evaluated by contour integration over the complex variable $k$ giving

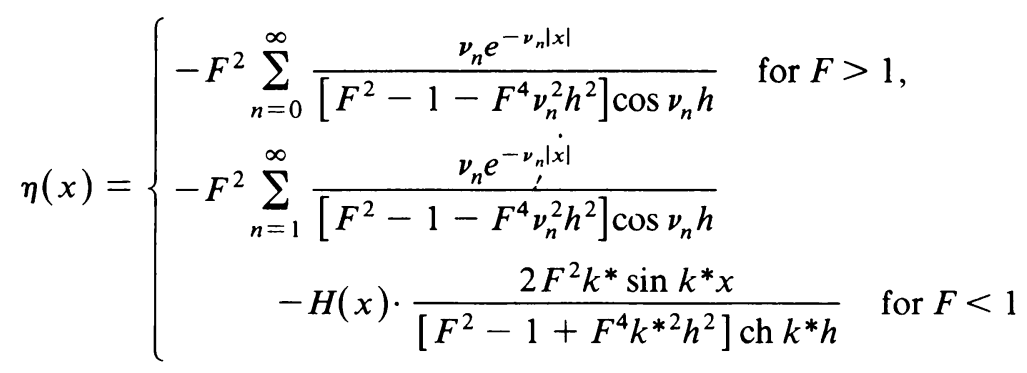

where

$$
H(x)= \begin{cases}1 & \text { for } x>0 \\ 0 & \text { for } x<0\end{cases}
$$

\footnotetext{
'The delta function bump solution exhibits the same qualitative features as more physical bumps and can easily be used to build up general solutions by superposition.
} 
and $i \nu_{n}, n=0,1,2, \ldots$, are the imaginary roots of $D(k)=0$ with lie in the upper half plane.

The shape of the linear free surface solution is distinctly different for $F>1$ as opposed to $F<1$. For $F>1, \eta(x)$ rises over the bump and is symmetric with respect to it. For $F<1, \eta(x)$ dips over the bump and is not symmetric with respect to it; waves are produced downstream. The free surface shapes are shown schematically in Figs. 2 and 3.

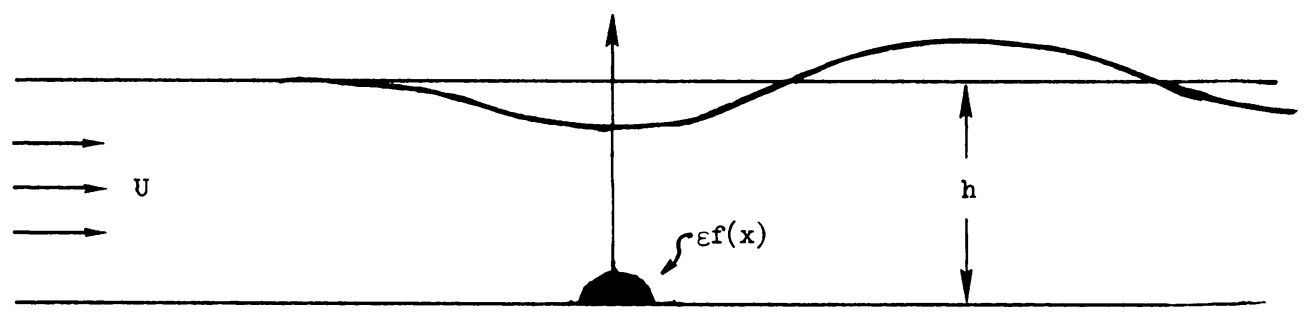

FIG. 2. Schematic representation of linear free surface for $F<1$.

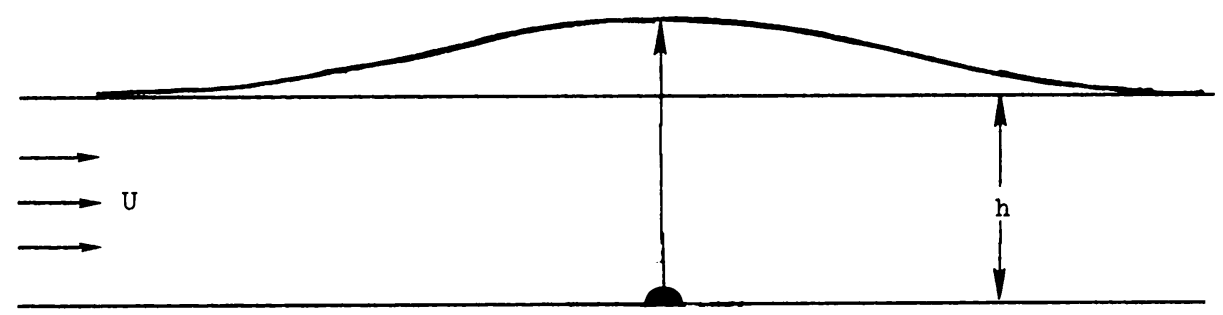

FIG. 3. Schematic representation of linear free surfaces for $F>1$.

Although the linear approximation is valid for any fixed $F$ not equal to 1 as long as $\varepsilon$ is taken small enough, it is not valid if $F$ is allowed to approach 1 . As $F$ tends to $1^{+}, \eta$ becomes unbounded near the bump,

$$
\eta \underset{F \rightarrow 1^{+}}{\sim} \frac{3}{2} \frac{e^{-\nu_{0}|x|}}{\nu_{0} h^{2}} \underset{\substack{F \rightarrow 1^{+}}}{\sim} O\left(F^{2}-1\right)^{-1 / 2} .
$$

Thus, the assumption that $\eta(x)$ is order 1 , which is inherent in the linear approximation, is not valid for $|x|$ near the bump as $F$ tends to $1^{+}$. As $F$ tends to $1^{-}, \eta(x)$ remains bounded upstream and near the bump but not far downstream

$$
\eta(x) \underset{\substack{F \rightarrow 1^{-} \\\left(F^{2}-1\right)^{1 / 2} x \ll 1}}{\sim} \frac{-3 x}{h^{2}} .
$$

A more exact description of the range of validity of the linear solution is given next through the second order theory.

3. The second order solution. The second order solution can be used to indicate the range of validity of the linear (first order) solution. The linear approximation assumes that the potential and free surface satisfy asymptotic expansions of the form 


$$
\Phi(x ; \varepsilon)=U\left\{x+\varepsilon \varphi(x, y)+\varepsilon^{2} \varphi_{2}(x, y)+\cdots\right\}
$$

and

$$
\xi(x ; \varepsilon)=\varepsilon \eta(x)+\varepsilon^{2} \eta_{2}(x)+\cdots
$$

where the functions $\varphi, \eta, \varphi_{2}, \eta_{2}$, etc. are order 1 . This idea can be generalized to include expansions of the same form where $\varphi, \eta, \varphi_{2}, \eta_{2}$, etc. are no longer order 1 but the terms $\varepsilon \varphi, \varepsilon^{2} \varphi_{2}, \ldots$ and $\varepsilon \eta, \varepsilon^{2} \eta_{2}, \ldots$ still appear in asymptotic order. In this sense, the linear approximation is still valid as $\varepsilon$ tends to zero as long as

$$
\frac{\Phi-[U(x-\varepsilon \varphi)]}{\varepsilon \varphi} \underset{\varepsilon \rightarrow 0}{\rightarrow 0} 0 \text { and } \frac{\xi-\varepsilon \eta}{\varepsilon \eta} \underset{\varepsilon \rightarrow 0}{\rightarrow} 0 .
$$

By solving for the second order terms, it is possible to determine when the second order terms and the first order terms are the same size and thus indicate when the linear theory is not valid.

Substituting the asymptotic expansions for $\Phi$ and $\xi$ into equations (1)-(4), expanding $\Phi$ in the boundary equations about $y=0$ or $y=h$, and retaining terms which are of order $\varepsilon$ gives the linear equations. The order $\varepsilon^{2}$ terms define the second order problem

$$
\begin{gathered}
\varphi_{2_{x x}}+\varphi_{2_{y y}}=0, \\
\varphi_{2 y}=\left(\varphi_{x} f\right)_{x} \text { on } y=0 \\
\varphi_{2 y}=-F^{2} h\left\{\varphi_{2_{x x}}+\left(\varphi_{x}^{2}+\varphi_{y}^{2}\right)_{x}\right\} \quad \text { on } y=h, \\
\frac{F^{2} h}{2}\left(\varphi_{x}^{2}+\varphi_{y}^{2}+2 \varphi_{2_{x}}\right)+F^{2} h \eta \varphi_{x y}+\eta_{2}=0 \text { on } y=h .
\end{gathered}
$$

The second order equations are somewhat more complicated than the linear equations. However, they are linear in the unknown functions $\varphi_{2}$ and $\eta_{2}$ ( $\varphi$ and $\eta$ are known) and can be solved exactly. The asymptotic results are merely quoted below.

For a delta function bump, $\eta_{2}$ (like $\eta$ ) becomes large near the bump as $F$ tends to $1^{+}$,

$$
\eta_{2} \underset{\left(F^{2}-1\right)^{1 / 2}|x| \ll 1}{\sim}-\frac{5}{4 h^{3}} \frac{1}{\left(F^{2}-1\right)^{2}}
$$

and as $F$ tends to $1^{-}$, remains bounded upstream and near the bump but not far downstream

$$
\eta_{2} \underset{\substack{F \rightarrow 1^{-} \\\left(F^{2}-1\right)^{1 / 2} x \ll 1}}{\sim}-\frac{9}{4 h^{7}} x^{4} .
$$

The linear approximation is not valid when the terms $\varepsilon^{2} \eta_{2}$ and $\varepsilon \eta$ are the same order. This never occurs for flows with $\left|F^{2}-1\right|>O\left(\varepsilon^{2 / 3}\right)$. For flows with

$$
F^{2}-1 \underset{\varepsilon \rightarrow 0}{\sim} O\left(\varepsilon^{2 / 3}\right),
$$

the terms $\varepsilon^{2} \eta_{2}$ and $\varepsilon \eta$ become the same order for supercritical flows when

$$
x \underset{\varepsilon \rightarrow 0}{\sim} O\left(\varepsilon^{-1 / 3}\right)
$$


upstream and for subcritical flows when

$$
x \underset{\varepsilon \rightarrow 0}{\sim} O\left(\varepsilon^{-1 / 3}\right)
$$

downstream. Thus, the subcritical linear solution appears valid for all $F$ (including $F=1^{-}$) from upstream infinity to a distance $O\left(\varepsilon^{-1 / 3}\right)$ downstream (see Fig. 4) while the supercritical flow for

$$
\left(F^{2}-1\right) \underset{\varepsilon \rightarrow 0}{\sim} O\left(\varepsilon^{2 / 3}\right)
$$

becomes nonlinear upstream.

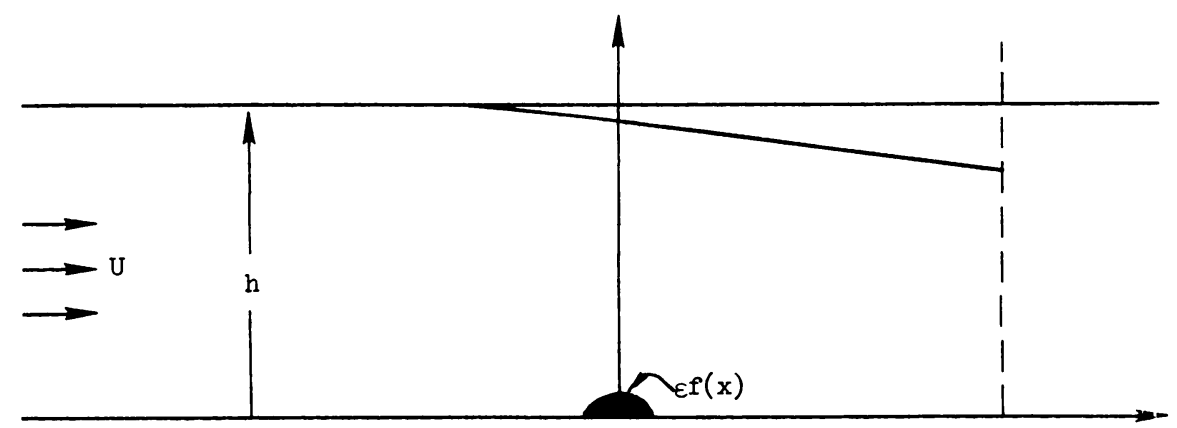

FIG. 4. Schematic representation of linear free surface for $F=1^{-}$.

In order for this problem to have a continuous solution when considered as a function of the parameter $F$, a nonlinear supercritical solution is required to match together the flow solutions near the bump as $F$ passes through 1 . This nonlinear solution must tend continuously to the linear solution for $F=1^{-}$as $F$ tends to $1^{+}$. The following nonlinear theory provides such a solution.

4. The nonlinear solution for $F>1$. The linear theory is based on the assumption that the basic incoming flow is uniform everywhere and that the bump produces only an order $\varepsilon$ perturbation to that flow. The following nonlinear theory is based on the assumption that the incoming flow is a (nonlinear) solitary wave.

The solitary wave potential and free surface are assumed to satisfy Cole's [2] asymptotic expansions of the form

$$
\begin{aligned}
\Phi_{S}=\left\{x+\varepsilon^{1 / 3}[\right. & f_{1}(\tilde{x})+\varepsilon^{2 / 3}\left(f_{2}(\tilde{x})-y^{2} / 2 f_{1}^{\prime \prime}(\tilde{x})\right) \\
& \left.\left.+\varepsilon^{4 / 3}\left(f_{3}(\tilde{x})-y^{2} / 2 f_{2}^{\prime \prime}(\tilde{x})+y^{4} / 4 ! f^{\prime \prime \prime \prime}(\tilde{x})\right)+\cdots\right]\right\}
\end{aligned}
$$

and

$$
\xi=h+\varepsilon^{2 / 3} \eta(\tilde{x})+\varepsilon^{4 / 3} \eta_{2}(\tilde{x})+\cdots
$$

where $\tilde{x}=\varepsilon^{1 / 3} x, F^{2}=1+\varepsilon^{2 / 3} K$ for $K>0$ with $\tilde{x}, K$ and the functions $\eta, f_{1}, \eta_{2}, f_{2}$, etc. and their $\tilde{x}$ derivatives order $1 .{ }^{2}$ These expansions reflect the fact that flow quantities in a

\footnotetext{
${ }^{2}$ The given solitary wave scaling is uniquely determined by the requirement that $\left(F^{2}-1\right) \underset{\varepsilon \rightarrow 0}{\sim} O\left(\varepsilon^{2 / 3}\right)$.
} 
solitary wave vary more slowly in the horizontal direction than the vertical direction.

Cole's solitary wave expansion for $\Phi_{s}$ and $\xi$ satisfy Laplace's equation and the kinematic boundary condition for flat a bottom for any $\eta, f_{1}, \eta_{2}, f_{2}$, etc. The relations defining $\eta, f_{1}$, $\eta_{2}, f_{2}$, etc. are found by substituting the expansions for $\Phi_{s}$ and $\xi$ into the free surface conditions and matching terms of equal order in $\varepsilon$. These relations uniquely define the incoming solitary wave in terms of the parameter $K$.

The resulting differential equation ${ }^{3}$ for $\eta$ is

$$
\frac{h^{3}}{3} \eta^{2}-h K \eta^{2}+\eta^{3}=0
$$

The function $f_{1}$ is defined in terms of $\eta$ as

$$
f_{1}^{\prime}=-\eta / h \text {. }
$$

Equation (13) defines a solitary wave centered about the bump with maximum height $h K$. The solitary wave solution is valid far upstream where the bottom is flat. Near the bump, it must be asymptotically matched onto a "local" solution which incorporates the boundary condition on the bump. Fortunately, the fact that flow characteristics in a solitary wave vary slowly in the horizontal direction in comparison to the vertical direction makes the desired matching easy.

As $\tilde{x}$ tends to zero from below, the solitary wave potential can be expanded in a Taylor series ib $x$,

$$
\Phi_{s}=U\left\{x+\varepsilon^{1 / 3} f_{1}(0)+\varepsilon^{2 / 3} x f_{1}^{\prime}(0)+\varepsilon\left[\left(\frac{x^{2}}{2}-\frac{y^{2}}{2}\right) f_{1}^{\prime \prime}(0)+f_{2}(0)\right]+o(\varepsilon)\right\}
$$

which is equivalent to

$$
\Phi_{s}=U\left(1-\varepsilon^{2 / 3} K\right)\left\{x+\varepsilon^{1 / 3} a+o(\varepsilon)\right\}
$$

where $a=f_{1}(0)\left(1+\varepsilon^{2 / 3} K\right)+\varepsilon^{2 / 3} f_{2}(0)$ is a constant. The term involving $f_{1}^{\prime \prime}(0)$ vanishes $^{4}$ since $f_{1}^{\prime \prime}(0)=-\eta(0) / h=0$. Near the bump, the fluid height is approximately $h\left(1+\varepsilon^{2 / 3} K\right)$ and the Froude number squared is

$$
F_{l}^{2} \underset{\varepsilon \rightarrow 0}{\sim} 1-2 \varepsilon^{2 / 3}
$$

(based on the local flow characteristics). This suggests that the upstream solitary wave solution can be asymptotically matched onto a "local" subcritical expansion for the linear problem with

$$
\Phi_{l}=U\left(1-\varepsilon^{2 / 3} K\right)\left\{x+\varepsilon^{1 / 3} a+\varepsilon \varphi(x, y)+\cdots\right\}
$$

and a local height $h_{l}=h\left(1+\varepsilon^{2 / 3} K\right)$. The solution for $\varphi(x, y)$ is given in Sec. 1 ; the presence of the constant term $\varepsilon^{1 / 3} a$ does not affect the flow characteristics since only derivatives of the potential enter into the problem.

\footnotetext{
${ }^{3}$ An arbitrary constant of integration in the differential equation for $\eta$ has been eliminated by also substituting the asymptotic expansions for $\Phi_{s}$ and $\xi$ into the mass flow equation $U h=\int_{0}^{h+\varepsilon^{2 / 3} \eta+\cdots} \Phi_{s_{x}} d y$ and matching terms of equal order in $\varepsilon$.

${ }^{4}$ In order for the solitary wave to match with the "local" solution, the solitary wave must be centered about the bump.
} 
Formally, matching is accomplished in the intermediate region by the variable $\hat{x}=\varepsilon^{\alpha} x$ where $0<\alpha<1 / 9$. Fixing $\hat{x}$ and letting $\varepsilon$ tend to zero gives

$$
x=\frac{\hat{x}}{\varepsilon^{\alpha}} \underset{\substack{\varepsilon \rightarrow 0 \\ \hat{x} \text { fixed }}}{\rightarrow}-\infty
$$

and

$$
\tilde{x}=\varepsilon^{1 / 3} x=\varepsilon^{1 / 3-\alpha} \hat{x} \underset{\substack{\varepsilon \rightarrow 0 \\ \hat{x} \text { fixed }}}{\rightarrow} 0 .
$$

Rewriting both potentials in terms of the variable $\hat{x}$ and letting $\varepsilon$ tend to zero gives

$$
\Phi_{s} \text { and } \Phi_{l} \underset{\varepsilon \rightarrow 0}{\sim} U\left(1-\varepsilon^{2 / 3} K\right)\left\{\frac{\hat{x}}{\varepsilon^{\alpha}}+\varepsilon^{1 / 3} a+o(\varepsilon)\right\} .
$$

These solutions indeed match asymptotically.

This composite solution predicts that the nonlinear supercritical flow with Froude number $F^{2}=1+\varepsilon^{2 / 3} K$ at upstream infinity rises along a solitary wave and passes over the bump with a local subcritical Froude number $F_{l}^{2}=1-2 \varepsilon^{2 / 3} K$. As $F$ tends to $1^{+}, K$ tends to zero and the maximum height of the solitary wave tends to zero. In this way, the local Froude number tends to $1^{-}$and the nonlinear solution tends continuously to the linear solution for $F=1^{-}$.

While Moiseev [3] and Fillipov [4] have postulated the possible nonuniqueness of supercritical solutions, these results do not support thei: hypothesis. The second order solution indicates that the linear theory is valid only for $F^{2}-1$ larger than $O\left(\varepsilon^{2 / 3}\right)$ while the above matching procedure requires $F^{2}-1$ to be $O\left(\varepsilon^{2 / 3}\right)$. Thus, the linear supercritical solution is valid only when the nonlinear solution is not and vice versa.

5. Concluding remarks. Even though the difficulties with the upstream supercritical flow appear to be resolved, all flows with

$$
F^{2}-1 \underset{\varepsilon \rightarrow 0}{\sim} O\left(\varepsilon^{2 / 3}\right)
$$

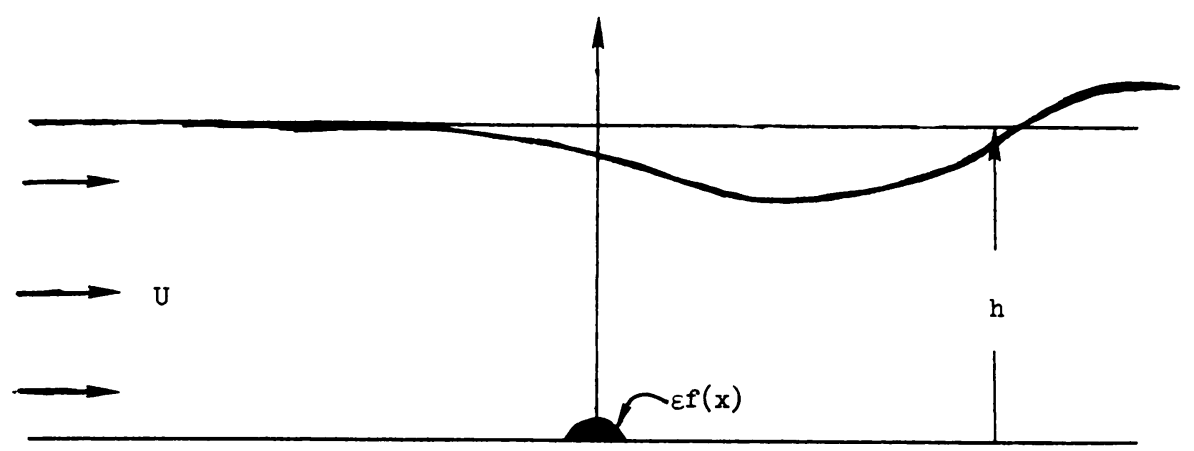

FIG. 5. Schematic representation of free surface for $F^{2}=1-\varepsilon^{2 / 3} K$ with $K>(81 / 4 h)^{1 / 3}$. 
become nonlinear for

$$
\underset{\varepsilon \rightarrow 0}{\sim} O\left(\varepsilon^{-1 / 3}\right)
$$

downstream.

Cole [2] has partially resolved this difficulty by matching the linear subcritical solutions for flows with $F^{2}=1-\varepsilon^{2 / 3} K$ and $K \geqslant\left(81 / 4 h^{4}\right)^{1 / 3}$ to cnoidal waves far downstream. The composite solution for one of these flows is given schematically in Fig. 5. This matching is not possible for smaller $K$.

\section{REFERENCES}

[1] . Kelvin, On stationary waves in flowing water, Mathematical and Physical Papers, Vol. IV, Cambridge Press, 270-302, 1910

[2] J. D. Cole, Limit process expansions and approximate equations, Singular Perturbations and Asymptotics, R. E. Meyer, S. Parter, ed., Academic Press, 35-39 1980

[3] N. N. Mosieev, On the Non-uniqueness of the Possible Forms of Steady Flows of a Heavy Fluid for Froude Numbers Close to 1, P.M.M. 21 860-864 (1957)

[4] I. G. Fillipov, Solution of the Problem of the Motion of a Vortex Under the Surface of a Fluid, for Froude Numbers Near Unity, P.M.M. 24, 478-490 (1960) 